\title{
Modular entanglement of atomic qubits using photons and phonons
}

\author{
D. Hucul ${ }^{\star}$, I. V. Inlek, G. Vittorini, C. Crocker, S. Debnath, S. M. Clark ${ }^{\dagger}$ and C. Monroe
}

Quantum entanglement is the central resource behind quantum information science, from quantum computation and simulation $^{1,2}$ to enhanced metrology ${ }^{3}$ and secure communication'. These applications require the quantum control of large networks of qubits to realize gains and speed increases over conventional devices. However, propagating entanglement becomes difficult or impossible as the system grows in size. Here, we demonstrate the first step in a modular approach ${ }^{4}$ to scaling entanglement by using complementary quantum buses on a collection of three atomic ion qubits stored in two remote ion trap modules. Entanglement within a module is achieved with deterministic near-field interactions through phonons $^{5}$, and remote entanglement between modules is achieved with a probabilistic interaction through photons ${ }^{6}$. This minimal system allows us to address generic issues in the synchronization of entanglement with multiple buses. It points the way towards a modular large-scale quantum information architecture that promises less spectral crowding and thus potentially less decoherence as the number of qubits increases ${ }^{4}$. We generate this modular entanglement faster than the observed remotely entangled qubit-decoherence rate, showing that entanglement can be scaled simply by adding more modules.

Small modules of qubits have been entangled through native local interactions in many physical platforms, such as trapped atomic ions through their Coulomb interaction ${ }^{5}$, Rydberg atoms through their electric dipoles ${ }^{7,8}$, nitrogen-vacancy centres in diamond through their magnetic dipoles ${ }^{9}$, and superconducting Josephson junctions through capacitive or inductive couplings ${ }^{10,11}$. However, each of these systems is confronted with practical limits to the number of qubits that can be reliably controlled, stemming from inhomogeneities, the complexity and density of the interactions between the qubits, or quantum decoherence. Scaling beyond these limits can be achieved by invoking a second type of interaction that can extend the entanglement to other similar qubit modules. Such an architecture should therefore exploit both the local interactions within the qubit modules, and also remote interactions between modules (an example architecture is shown in Fig. 1). One promising approach is to directly move qubits between different modules $^{12,13}$, but this approach is limited by the difficulty of moving qubits over large distances. Optical interfaces provide ideal buses for extending entanglement between modules ${ }^{14,15}$, as optical photons can propagate over macroscopic distances with negligible loss. Several qubit systems have been entangled through remote optical buses, such as atomic ions ${ }^{16}$, neutral atoms ${ }^{17}$ and nitrogen-vacancy centres in diamond ${ }^{18}$.
In the experiment reported here, we juxtapose local phonon and remote photon entanglement buses using trapped atomic ion qubits, balancing the requirements of each interface within the same qubit system. The observed entanglement rate within and between modules is faster than the observed entangled qubitdecoherence rate. This is critical in quantum modular architectures because the required resource scaling is superexponential in the ratio of decoherence rate to entanglement rate ${ }^{4}$. This ratio is observed to be 0.2 in this experiment, many orders of magnitude lower than previous experiments demonstrating remote entanglement ${ }^{17-19}$. Overcoming the resource scaling requirement makes trapped ions a leading candidate for realizing a quantum network.

The qubits in this experiment are defined by the two hyperfine 'clock' states, $\left|F=0, m_{F}=0\right\rangle \equiv|0\rangle$ and $\left|F=1, m_{F}=0\right\rangle \equiv|1\rangle$, which are separated by $\omega_{0}=2 \pi \times 12.64282 \mathrm{GHz}$ in the ${ }^{2} S_{1 / 2}$ manifold of trapped ${ }^{171} \mathrm{Yb}^{+}$atoms. Laser cooling, optical pumping, and readout occur via standard state-dependent fluorescence techniques ${ }^{20}$. The qubits are trapped in two independent modules separated by $\sim 1 \mathrm{~m}$, as shown in Fig. 1a. (The ion traps, light collection optics and interferometer could in principle be part of a modular, scalable architecture, as shown in Fig. 1b.)

To generate remote entanglement between atoms in physically separated ion trap modules, we synchronously excite each atom with a resonant fast laser pulse ${ }^{16}$. A fraction of the resulting spontaneously emitted light is collected into an optical fibre, with each photon's polarization $\left(\sigma^{+}\right.$or $\left.\sigma^{-}\right)$entangled with its parent atom owing to atomic selection rules (Fig. 2a). Each photon passes through a quarter-wave plate that maps circular to linear polarization $\left(\sigma^{+} \rightarrow H\right.$ and $\sigma^{-} \rightarrow V$ ), and then the two photons interfere on a 50/50 beamsplitter, where detectors monitor the output (see Fig. 1a and Methods) ${ }^{19}$. We select the two-photon Bell states of light $|H V\rangle+\mathrm{e}^{i \phi_{\mathrm{D}}}|V H\rangle$, where $\phi_{\mathrm{D}}$ is 0 or $\pi$ depending on which pair of detectors registers the photons ${ }^{21}$. Finally, a series of microwave pulses transfers the atoms into the $\{|0\rangle,|1\rangle\}$ basis (Fig. 2b), ideally resulting in the heralded entangled state of the two remote atomic qubits $|01\rangle+\mathrm{e}^{i \phi_{\mathrm{AB}}}|10\rangle$.

The intermodular phase is given by

$$
\phi_{\mathrm{AB}}=\phi_{\mathrm{D}}+\Delta \omega_{\mathrm{AB}} t+k c \Delta \tau+k \Delta x+\Delta \phi_{\mathrm{T}}
$$

In this equation, the phase evolves with the difference in qubit splittings between module $\mathrm{A}$ and $\mathrm{B}$, $\Delta \omega_{\mathrm{AB}}=\omega_{0, \mathrm{~A}}-\omega_{0, \mathrm{~B}} \approx 2 \pi \times 2.5 \mathrm{kHz}$, owing to controlled Zeeman shifts $^{20}$. The stable geometric phase factors $k c \Delta \tau<10^{-2}$ and $k \Delta x<10^{-2}$ result from the difference in excitation time $\Delta \tau<100$ 
a

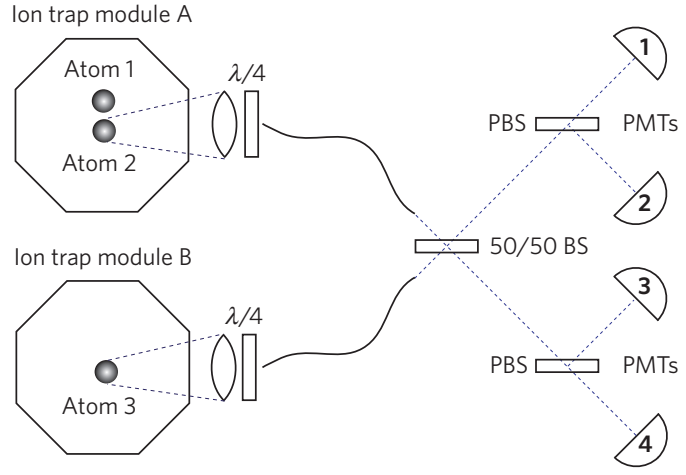

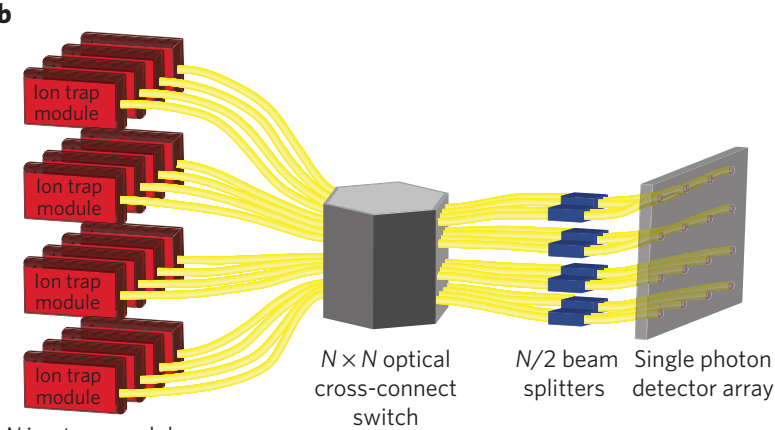

Figure 1 | Experimental set-up and a modular architecture for a large-scale quantum network. a, Two modules separated by $\sim 1 \mathrm{~m}$ each contain an ion trap. High numerical aperture objectives couple spontaneously emitted photons from a single atom into a single-mode optical fibre. The photons from atoms in separate traps interfere on a 50/50 beamsplitter (BS), are sorted by polarizing beamsplitters (PBS), then detected by photomultiplier tubes (PMTs). Coincident detection of photons on specific PMT pairs heralds entanglement of atomic spins (Methods). b, Schematic of a large-scale, modular quantum network of trapped ions. Ion trap modules (red boxes) confine atoms coupled together through their Coulomb bus, and entanglement within modules is accomplished with the application of spin-dependent forces on the trapped atoms ${ }^{4}$. Probabilistic, heralded entanglement is generated between modules via interference of emitted photons from each module. A reconfigurable $N \times N$ cross-connect switch links arbitrary modules. Photon interference occurs at fibre beamsplitters, and a single-photon detector array heralds entanglement of atomic spins between modules.

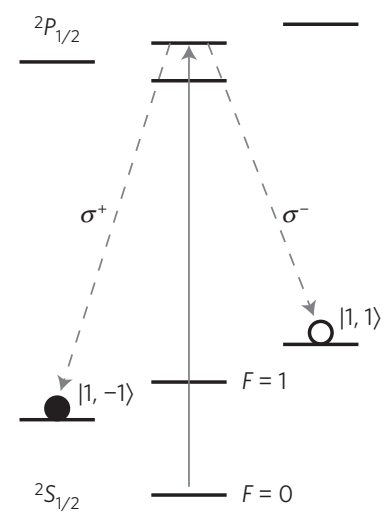

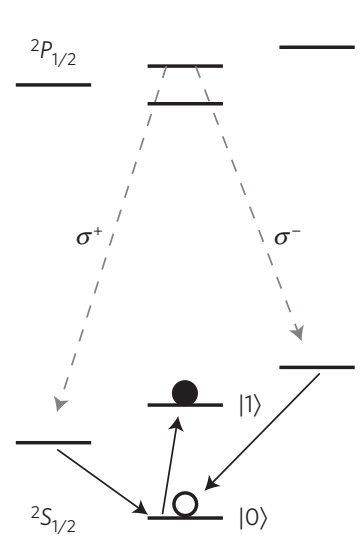

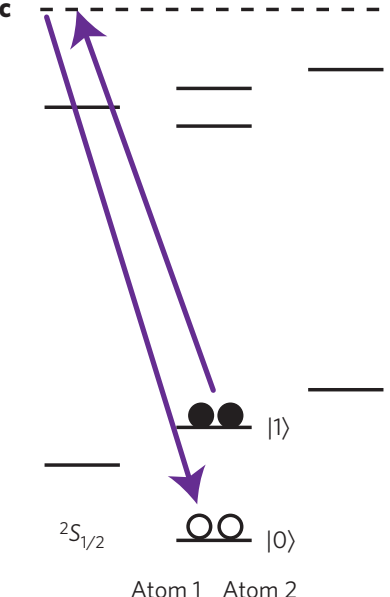

Figure 2 | Qubit manipulations for generating entanglement between and within modules. a, Resonant excitation scheme and single-photon emission in an $\mathrm{Yb}^{+}$atom system. After optically pumping the atoms to the $\left|F, m_{F}\right\rangle=|0,0\rangle$ state of the ${ }^{2} S_{1 / 2}$ manifold, a frequency-doubled, mode-locked Ti:sapphire laser excites the atom to the $|1,0\rangle$ state of the ${ }^{2} P_{1 / 2}$ manifold, whereby the atom decays to the $|1, \pm 1\rangle$ states via emission of $\sigma^{\mp}$ polarized photons into optical fibres. b. After interference of the two photons on a 50/50 non-polarizing beamsplitter, we apply a series of microwave transfer pulses to transfer the entangled state to the clock basis, resulting in the state $|01\rangle+\mathrm{e}^{i \phi_{A B}}|10\rangle$, where $\phi_{A B}$ is the intermodular phase. c, We entangle atomic spins within module A through spin-dependent optical dipole forces using off-resonant lasers (purple arrows) ${ }^{5,23}$.

ps and difference in path length $\Delta x<3 \mathrm{~cm}$ between each atom and the beamsplitter. Here $c$ is the speed of light and $k \sim 0.33 \mathrm{~m}^{-1}$ is the wavenumber associated with the energy difference of the photon decay modes (here, the energy difference between $\sigma^{+}$and $\sigma^{-}$photons). The final contribution is the stable phase difference of the microwave transfer pulses $\Delta \phi_{\mathrm{T}}$ across the modules.

In previous experiments, entanglement between remote atom spins at rates of $0.002 \mathrm{~s}^{-1}$ was accomplished using atom-photon frequency entanglement ${ }^{22}$, and at rates of $0.026 \mathrm{~s}^{-1}$ using atomphoton polarization entanglement ${ }^{19}$. Here, we greatly increase the single-photon collection efficiency by using high numerical aperture microscope objectives and detecting two out of four Bell states of light emitted by the atoms to achieve a heralded entanglement rate of $4.5 \mathrm{~s}^{-1}$ (Methods).

Given a heralded photon coincidence event, we verify entanglement between ion trap modules by measuring atomic state populations and coherences following standard two-qubit tomography protocols ${ }^{23}$. We measure an average entangled Bell state fidelity of $0.78 \pm 0.03$. Imperfect mode matching at the beamsplitter contributes $0.08 \pm 0.02$ to the infidelity. The measured atom-photon polarization entanglement is 0.92 per ion trap, which contributes 0.15 to the remote entangled state infidelity. We attribute the atom-photon polarization infidelity to spatially inhomogeneous rotations of the photon polarization, polarizationdependent loss, and multiple excitations of the remote atoms from imperfect pulse picking of resonant fast laser pulses. Combining imperfect ion-photon polarization entanglement with imperfect mode matching at the beamsplitter yields an expected fidelity of $0.79 \pm 0.02$, consistent with observation. This fidelity could be improved with the use of fibre beamsplitters to improve spatial mode matching of the photons. In addition, the use of phase masks could correct polarization error introduced by optical elements associated with single-photon collection and transmission. Electrooptic pulse pickers with higher extinction ratios could reduce 
a

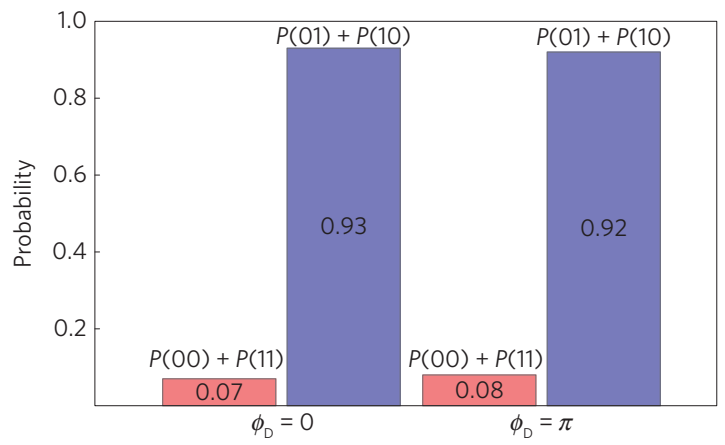

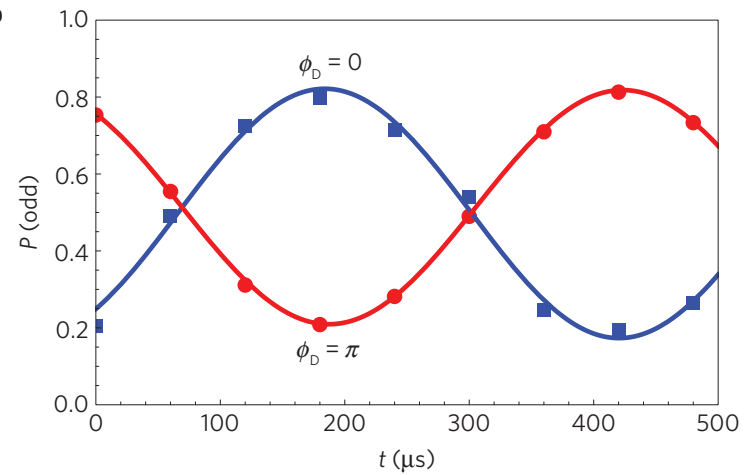

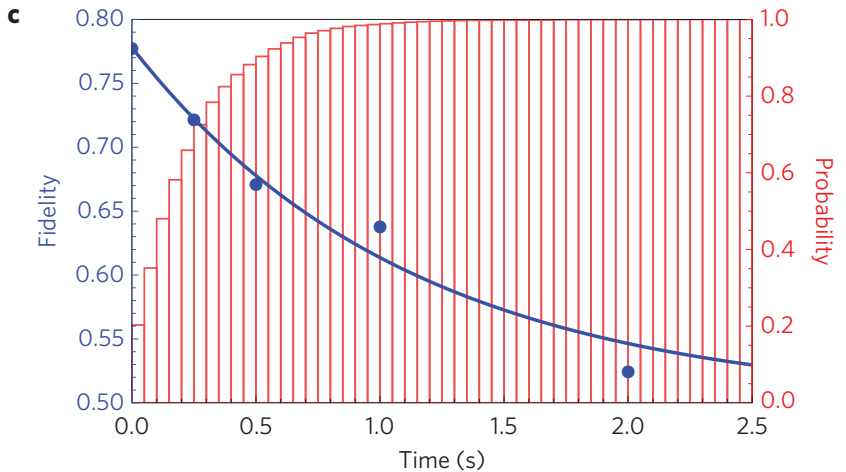

Figure 3 | Heralded entanglement fidelity and rate between modules. a, Populations of two remote atoms after heralding entanglement between modules. After detecting the photon Bell states $\left(\phi_{D}=0\right.$ or $\pi$ ), microwave transfer pulses rotate the remote atom populations to the $\{|0\rangle,|1\rangle\}$ basis. Subsequent detection of the remotely entangled atoms results in measurement of odd parity, $P(01)+P(10)$, with high probability. $\mathbf{b}$, Phase coherent time evolution of the remote entangled state with the application of an intermodular magnetic field gradient. After heralding remote entanglement between modules and applying microwave transfer pulses, the addition of a time delay before a $\pi / 2$ rotation on both atoms results in an out-of-phase oscillatory behaviour of the remote atom entangled state with $\phi_{D}=0$ or $\pi$ (blue squares and red circles respectively, see equation (1)). c, Remote entangled state coherence and generation probability versus time. We measure the remote entangled state coherence time by adding a Ramsey zone delay in the presence of an intermodular magnetic field gradient before application of a spin-echo pulse and a $\pi / 2$ microwave rotation, as described in the text. The decay of the fidelity from the measured loss of phase coherence of the entangled state points to magnetic field gradient noise as the dephasing mechanism. A fit to an exponential function yields a coherence time of $1.12 \mathrm{~s}$. The probability of generating entanglement after a given time interval is shown in red. A fit to an exponential function gives the average remote entanglement rate $4.5 \mathrm{~s}^{-1}$. Error bars in a-c (not shown for clarity) are $\pm 1.4 \%$ due to state detection error (Methods).

errors associated with multiple excitations of remote atoms in different modules.

Because the phase of the entangled state evolves in time (second term of equation (1)), the remote atomic entanglement coherence time can be measured with Ramsey spectroscopy. Unlike a Ramsey experiment with a single atom, this measurement is not sensitive to long-term stability of the local oscillator ${ }^{20,24}$. We measure the remote entangled state coherence time by repeating the above experiment with constant transfer pulse phase $\Delta \phi_{\mathrm{T}}$ while varying the Ramsey zone delay before a final $\pi / 2$ microwave rotation. We use a spinecho pulse in the middle of the Ramsey zone delay to account for slow magnetic field gradient drifts, and measure an entanglement coherence time of $1.12 \mathrm{~s}$, well in excess of the required time to create remote entanglement between modules (Fig. 3c). Our experiment thus crosses the threshold where fault-tolerant error correction can propagate entanglement without a superexponential overhead in resources ${ }^{4}$.

In addition to using a photonic interconnect between ion traps, we use the Coulomb-coupled transverse phonon modes of the atoms to create entanglement within one module (Fig. 2c). Off-resonant laser beams drive stimulated Raman transitions between the qubit levels and impart spin-dependent forces detuned from the phonon modes. Following conventional Coulomb gate protocols ${ }^{5,25}$, after a certain time the motion returns to its original state (Methods), and the four two-qubit basis states are ideally mapped to the following entangled states

$$
\begin{array}{rlrl}
|00\rangle & \rightarrow|00\rangle-i \mathrm{e}^{-i \phi_{\mathrm{A}}}|11\rangle & |01\rangle \rightarrow|01\rangle-i|10\rangle \\
|11\rangle \rightarrow|11\rangle-i \mathrm{e}^{i \phi_{\mathrm{A}}}|00\rangle & |10\rangle \rightarrow|10\rangle-i|01\rangle
\end{array}
$$

where $\phi_{\mathrm{A}}$ is the intramodular phase from this optical Raman process in module A (ref. 26). This phase depends on the relative optical phase of two non-copropagating lasers. Using the above gate operation on two Doppler-cooled atoms within a module $(\bar{n} \sim 3)$, we create the state $|00\rangle-i \mathrm{e}^{-i \phi_{\mathrm{A}}}|11\rangle$ with a fidelity of $0.85 \pm 0.01$, excluding detection error, as shown in Fig. 4a,b. Cooling below the Doppler limit was not implemented in this experiment to keep the experimental repetition rate high for fast generation of remote entanglement. Higher-fidelity Coulomb gates may be achieved by better control of the RF amplitude applied to the ion trap and through the use of ground state cooling to reduce sensitivity to small detuning errors from the trapped atoms' sidebands of motion.

We now describe the integration of both photonic and phononic buses to generate entangled three-particle states. The three atoms are first prepared in the state $\left|\psi_{1} \psi_{2}\right\rangle_{\mathrm{A}}\left|\psi_{3}\right\rangle_{\mathrm{B}}=|00\rangle_{\mathrm{A}}|0\rangle_{\mathrm{B}}$, with atoms 1 and 2 in module A and the remote atom 3 in module B (Fig. 1a). 


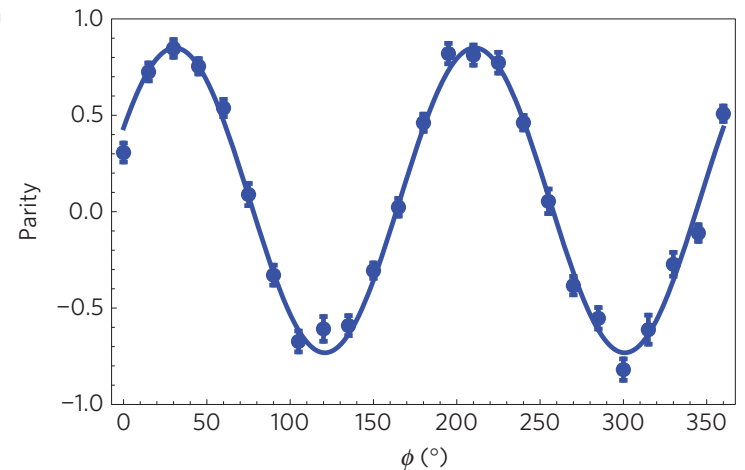

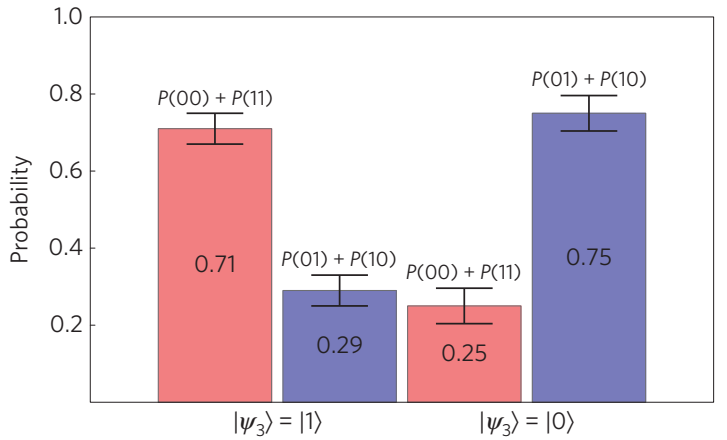

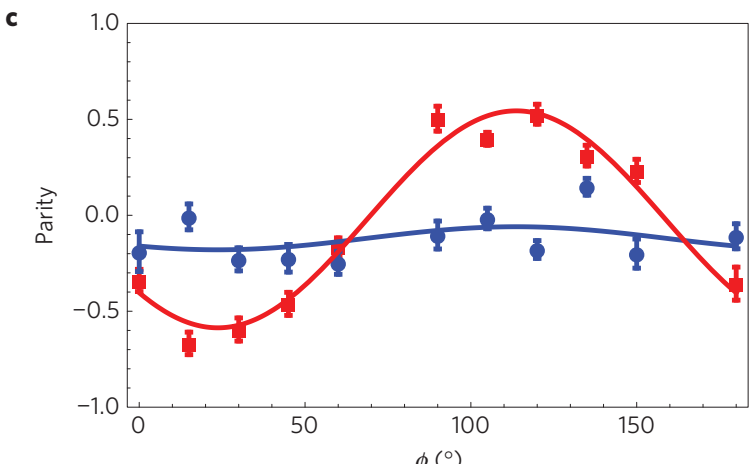

Figure 4 | Entanglement between qubits in the same module without and with heralded entanglement between modules. a, After preparing the atoms in the state $|00\rangle$ and applying an entangling gate through phonons within a module (equation (2)), we measure the parity of the entangled state following

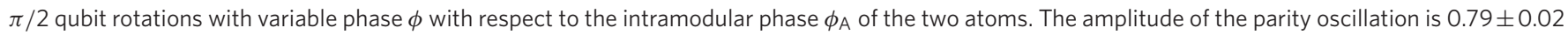
and the fidelity of the entangled state is $0.85 \pm 0.01$ excluding state detection errors. $\mathbf{b}$, Populations of two atoms in ion trap module $A$ after remote entanglement between atoms 2 and 3 followed by entanglement between atoms 1 and 2, as described in the text. After measuring the resulting three-particle state (equation (3)), if the remote atom is in the state $|1\rangle$, atoms 1 and 2 should be in an even parity state. If the remote atom is in the state $|0\rangle$, atoms 1 and 2 should be in an odd parity state. We observe this correlation with the remote atom with probability $0.71 \pm 0.04$ and $0.75 \pm 0.05$, respectively, after averaging over detection of the entangled photon states. c, Parity oscillation of atoms 1 and 2 conditioned on detecting the remote atom in the state $|1\rangle_{B}$ (red squares) and $|0\rangle_{B}$ (blue circles). After remote entanglement between modules and entanglement within one module, we apply a Raman $\pi / 2$ rotation with variable phase $\phi$ to atoms 1 and 2 in module $A$ and measure the state of all three atoms. If the remote atom is in the state $|0\rangle_{B}$, a

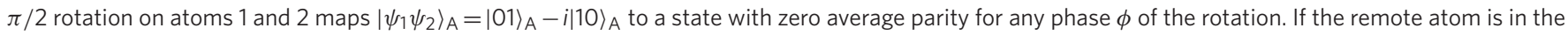
state $|1\rangle_{B}$, a $\pi / 2$ rotation with variable phase $\phi$ of $\left|\psi_{1} \psi_{2}\right\rangle_{A}=|00\rangle_{A}-i e^{-\phi_{A}}|11\rangle_{A}$ maps the parity of this state to cos $\left(\phi_{A}-2 \phi\right)$. We observe such a parity oscillation correlated with the state of the remote atom. The fidelity of the two-qubit entangled state $|00\rangle_{A}-i e^{-i \phi_{A}}|11\rangle_{A} c^{c o n d i t i o n e d ~ o n ~ d e t e c t i n g ~ t h e ~}$ remote atom in $\left|\psi_{3}\right\rangle_{B}=|1\rangle_{B}$ is $0.63 \pm 0.03$. Error bars in a-c are the fit error of experimental histograms of the two qubits' four basis states.

After heralding entanglement between atom 2 in module A and atom 3 in module $B$ using photons, we re-initialize atom 1 to the state $|0\rangle_{\mathrm{A}}$ with an individual addressing optical pumping beam, and then we entangle atoms 1 and 2 within module A using phonons. Ideally, this produces the state

$$
\begin{aligned}
\left|\psi_{1} \psi_{2}\right\rangle_{\mathrm{A}}\left|\psi_{3}\right\rangle_{\mathrm{B}}= & \left(|00\rangle_{\mathrm{A}}-i \mathrm{e}^{-i \phi_{\mathrm{A}}}|11\rangle_{\mathrm{A}}\right)|1\rangle_{\mathrm{B}} \\
& +\mathrm{e}^{i \phi_{\mathrm{AB}}}\left(|01\rangle_{\mathrm{A}}-i|10\rangle_{\mathrm{A}}\right)|0\rangle_{\mathrm{B}}
\end{aligned}
$$

In the above state, the parity of any pair of atoms is correlated with the spin state of the third atom. We take advantage of this property to probe the parity of atoms 1 and 2 in module A, and correlate it with the state of remote atom 3 in module B. After making photon and phonon connections between the atoms, we apply a $\pi / 2$ Raman rotation to atoms 1 and 2 with a variable phase $\phi$, followed by state detection of all three atoms. When the remote atom is measured in state $\left|\psi_{3}\right\rangle_{\mathrm{B}}=|1\rangle$, the spin parity of atoms 1 and 2 in module $\mathrm{A}$ is $\Pi=\Pi_{c} \cos \left(\phi_{\mathrm{A}}-2 \phi\right)$. When the remote atom is measured in state $\left|\psi_{3}\right\rangle=|0\rangle_{\mathrm{B}}$, the atoms in module $\mathrm{A}$ should be mapped to a state with zero average parity, regardless of the phase of the $\pi / 2$ Raman rotation. We observe this correlation with a remote entangled state generation rate of $\sim 4 \mathrm{~s}^{-1}$, as shown in Fig. $4 \mathrm{~b}$,c. The fidelity of detecting the state $|00\rangle_{\mathrm{A}}-i \mathrm{e}^{-i \phi_{\mathrm{A}}}|11\rangle_{\mathrm{A}}$ of atoms 1 and 2 conditioned on detecting the remote atom 3 in the state $|1\rangle_{\mathrm{B}}$ is $0.63 \pm 0.03$.

Scaling this architecture to many modules can vastly simplify the complexity of phases to be tracked and controlled. For $N \gg 1$ modules each with $n \gg 1$ qubits and $m \ll n$ optical ports at each module, the number of overall phases is reduced by a factor of $1 / N+(m / n)^{2}$ compared to that for a fully connected set of $n N$ qubits ${ }^{4}$. Of course, in a modular architecture there may be overheads associated with the reduced connectivity, but it will be useful to have flexibility in this trade-off.

The intermodular phase $\phi_{\mathrm{AB}}$ in the experiment is easily controlled by setting the phase difference of microwave rotations between the two modules. The intramodular phase $\phi_{\mathrm{A}}$ is determined by the optical phase difference of the two Raman lasers and is passively stable for a single entangling experiment for typical gate times of the order of $100 \mu$ s. Tracking and controlling the optical phases between many entangled pairs in spatially separated modules at different times can be accomplished by using 'phase insensitive' gates ${ }^{26}$. All phases introduced by qubit rotations and entangling operations 
between and within modules can be referenced to a common, highquality master oscillator ${ }^{27}$

Scaling this system will also require mitigating crosstalk within modules. For example, when generating photons for intermodular entanglement, laser scatter and radiated light will disturb neighbouring qubits within a module. This may require the use of different species of atoms as photonic and memory qubits. Quantum information could then be transferred from the photonic qubits to the memory qubits via the Coulomb bus ${ }^{28}$. The second (photonic) species can also be used for intermittent sympathetic cooling ${ }^{29}$.

The modular architecture demonstrated in this experiment can be expanded to include many modules. Here an optical cross-connect switch can create a flexible, reconfigurable photonic network between modules (Fig. 1b) and thus be made fault tolerant for the execution of extended quantum circuits ${ }^{4}$. Modular architectures may be used as the backbone of a quantum repeater network ${ }^{30}$ and of a quantum network of clocks $^{31}$. The distance between nodes may be increased with the development of low-loss ultraviolet fibres or the efficient down-conversion of photons to telecommunication wavelengths, without affecting the entanglement rate, enabling long-distance quantum networks ${ }^{32}$.

\section{Methods}

In this experiment, ion trap module $\mathrm{A}$ is a segmented, four-blade design useful for holding chains of trapped atoms. A trap drive frequency of $37.15 \mathrm{MHz}$ is used to achieve secular transverse frequencies of $\sim 2.4 \mathrm{MHz}$. Module B is a four-rod Paul trap that confines a single atom. This trap is driven at $37.72 \mathrm{MHz}$ to achieve secular frequencies of $\sim 1.5 \mathrm{MHz}$.

To generate remote entanglement between atoms in physically separated ion traps, we optically pump both atoms to the $|0,0\rangle$ state. A picosecond laser pulse resonant with the ${ }^{2} S_{1 / 2} \rightarrow{ }^{2} P_{1 / 2}$ transition excites trapped atoms in different modules. The atoms spontaneously emit photons, of which $\sim 10 \%$ are collected by a large NA $=0.6$ single-atom microscope objective, resulting in the entangled photon-polarization, atom-spin state $1 / 2\left(|1,1\rangle\left|\sigma^{-}\right\rangle-|1,-1\rangle\left|\sigma^{+}\right\rangle\right)^{\otimes 2}$. The emitted photons pass through quarter-wave plates to convert the photon polarization to linear horizontal $(\mathrm{H})$ or linear vertical $(\mathrm{V})$, resulting in the atom-photon state $(|1,1\rangle|V\rangle-i|1,-1\rangle|H\rangle)^{\otimes 2}$. Each objective is mode matched to a single-mode optical fibre which delivers the photons to an interferometer with a 50/50 beamsplitter as the central element. The interferometer effects a Bell state measurement of the photon state. We detect two out of the four possible Bell states of light exiting the beamsplitter to herald the entanglement of the remote atoms' spins ${ }^{21}$; after a series of microwave transfer pulses, the remote atom entangled state is $|01\rangle+\mathrm{e}^{i \phi_{A B}}|10\rangle$, with the intermodular phase $\phi_{\mathrm{AB}}$ defined in the main text. The phase $\phi_{\mathrm{D}}$ is 0 if coincident photons are detected on PMTs 1 and 2 or 3 and 4 (Fig. 1a). The phase $\phi_{\mathrm{D}}$ is $\pi$ if coincident photons are detected on PMTs 1 and 3 or 2 and 4 .

The remote entanglement rate is limited by the collection and detection efficiency of emitted photons from the atoms. The probability for coincident detection of two emitted photons on exciting both atoms simultaneously with a resonant laser pulse is $P=p_{\text {Bell }}\left[P_{\pi} P_{S_{1 / 2}} Q_{\mathrm{E}} T_{\mathrm{fib}} T_{\mathrm{opt}}(\Omega / 4 \pi)\right]^{2}=9.7 \times 10^{-6}$, where $P_{\pi}=0.95$ is the probability of exciting the atom with a resonant ${ }^{2} S_{1 / 2} \rightarrow{ }^{2} P_{1 / 2}$ laser pulse, $P_{S_{1 / 2}}=0.995$ is the probability to decay from ${ }^{2} P_{1 / 2} \rightarrow{ }^{2} S_{1 / 2}$ (as opposed to the ${ }^{2} D_{3 / 2}$ state), $p_{\text {Bell }}=1 / 2$ accounts for selecting two of the four possible Bell states of light, $Q_{\mathrm{E}} \approx 0.35$ is the quantum efficiency of the single-photon PMT detectors, $T_{\text {fib }} \approx 0.14$ is the fibre coupling and transmission probability of a single-mode optical fibre, $T_{\text {opt }}=0.95$ is the photon transmission through optical components and $(\Omega / 4 \pi)=0.1$ is the fraction of the solid angle each microscope objective subtends. The experimental repetition rate of $470 \mathrm{kHz}$ is limited by the need for Doppler cooling (adding $\sim 500 \mathrm{~ns}$ on average to the repetition time), the atomic state lifetime of the ${ }^{2} P_{1 / 2}$ state (necessitating $\sim 1 \mu$ s of optical pumping for state preparation of the pure quantum state $|0\rangle)$, and sound wave propagation time in the acousto-optic modulator crystals used in the experiment. These factors result in a measured atom-atom entanglement rate of $4.5 \mathrm{~s}^{-1}$.

The Coulomb entangling gate makes use of Walsh function modulation $W[1]$ to reduce the sensitivity of the gate to detuning and timing errors ${ }^{33}$. We pick a detuning $\delta$ from a transverse mode of motion and set the gate time $t_{\mathrm{g}}=2 / \delta$ with a $\pi$ phase advance of the sidebands at $t=t_{\mathrm{g}} / 2$. We adjust the average Raman laser intensity power to make the sideband Rabi frequency $\eta \Omega$ satisfy $\delta=2^{3 / 2} \eta \Omega$ to complete the entangling gate $|00\rangle \rightarrow|00\rangle-i \mathrm{e}^{-i \phi_{\mathrm{A}}}|11\rangle$ in ion trap module A.
Detection error of a single atom in an ion trap module is limited by off-resonant pumping from the $F=1$ to the $F=0$ manifold of the ${ }^{2} S_{1 / 2}$ ground state through the $F=1$ manifold of the ${ }^{2} P_{1 / 2}$ excited state ${ }^{20}-$ it is $\sim 1 \%$ in the experiments presented here. Detection error of two qubits in the same module is limited by the use of a single PMT detector, where the photon detection histograms of a single qubit in the state $|1\rangle$ and two qubits in the state $|11\rangle$ may overlap. This overlap is $\sim 8 \%$ in these experiments.

Received 10 July 2014; accepted 9 October 2014; published online 17 November 2014

\section{References}

1. Nielsen, M. A. \& Chuang, I. L. Quantum Computation and Quantum Information (Cambridge Univ. Press, 2000).

2. Quantum simulation. Nature Phys. 8, (Insight issue) 263-299 (2012).

3. Giovannetti, V., Lloyd, S. \& Maccone, L. Quantum metrology. Phys. Rev. Lett. 96, 010401 (2006).

4. Monroe, C. et al. Large scale modular quantum computer architecture with atomic memory and photonic interconnects. Phys. Rev. A 89, 022317 (2014).

5. Blatt, R. \& Wineland, D. J. Entangled states of trapped atomic ions. Nature 453, 1008-1015 (2008).

6. Duan, L-M. \& Monroe, C. Colloquium: Quantum networks with trapped ions. Rev. Mod. Phys. 82, 1209-1224 (2010).

7. Isenhower, L. et al. Demonstration of a neutral atom controlled-NOT quantum gate. Phys. Rev. Lett. 104, 010503 (2010).

8. Wilk, T. et al. Entanglement of two individual neutral atoms using Rydberg blockade. Phys. Rev. Lett. 104, 010502 (2010).

9. Dolde, F. et al. Room-temperature entanglement between single defect spins in diamond. Nature Phys. 9, 139-143 (2013).

10. Dicarlo, L. et al. Preparation and measurement of three-qubit entanglement in a superconducting circuit. Nature 467, 574-578 (2010).

11. Neeley, M. et al. Generation of three-qubit entangled states using superconducting phase qubits. Nature 467, 570-573 (2010).

12. Kielpinski, D., Monroe, C. \& Wineland, D. J. Architecture for a large-scale ion-trap quantum computer. Nature 417, 709-711 (2002).

13. Barrett, M. et al. Deterministic quantum teleportation of atomic qubits. Nature 429, 737-739 (2004)

14. Cirac, J. I., Zoller, P., Kimble, H. J. \& Mabuchi, H. Quantum state transfer and entanglement distribution among distant nodes in a quantum network. Phys. Rev. Lett. 78, 3221-3224 (1997).

15. Duan, L-M., Lukin, M. D., Cirac, J. I. \& Zoller, P. Long-distance quantum communication with atomic ensembles and linear optics. Nature $\mathbf{4 1 4}$, 413-418 (2001).

16. Moehring, D. L. et al. Entanglement of single-atom quantum bits at a distance. Nature 449, 68-71 (2007).

17. Nölleke, C. et al. Efficient teleportation between remote single-atom quantum memories. Phys. Rev. Lett. 110, 140403 (2013).

18. Pfaff, W. et al. Unconditional quantum teleportation between distant solid-state quantum bits. Science 345, 532-535 (2014).

19. Matsukevich, D. N., Maunz, P., Moehring, D. L., Olmschenk, S. \& Monroe, C. Bell inequality violation with two remote atomic qubits. Phys. Rev. Lett. 100, 150404 (2008)

20. Olmschenk, S. et al. Manipulation and detection of a trapped $\mathrm{Yb}^{+}$hyperfine qubit. Phys. Rev. A 76, 052314 (2007).

21. Simon, C. \& Irvine, W. T. M. Robust long-distance entanglement and a loophole-free Bell test with ions and photons. Phys. Rev. Lett. 91, 110405 (2003).

22. Olmschenk, S. et al. Quantum teleportation between distant matter qubits. Science 323, 486-489 (2009).

23. Sackett, C. A. et al. Experimental entanglement of four particles. Nature 404, 256-259 (2000).

24. Chwalla, M. et al. Precision spectroscopy with two correlated atoms. Appl. Phys. B 89, 483-488 (2007).

25. Mølmer, K. \& Sørensen, A. Entanglement and quantum computation with ions in thermal motion. Phys. Rev. A 62, 022311 (1999).

26. Lee, P. J. et al. Phase control of trapped ion quantum gates. J. Opt. B 7, S371-S383 (2005).

27. Inlek, I. V., Vittorini, G., Hucul, D., Crocker, C. \& Monroe, C. Quantum gates with phase stability over space and time. Phys. Rev. A 90, 042316 (2014)

28. Schmidt, P. O. et al. Spectroscopy using quantum logic. Science 309, 749-752 (2005).

29. Barret, M. D. et al. Sympathetic cooling of ${ }^{9} \mathrm{Be}^{+}$and ${ }^{24} \mathrm{Mg}^{+}$for quantum logic. Phys. Rev. A 68, 042302 (2003). 
30. Briegel, H-J., Dürr, W., Cirac, J. I. \& Zoller, P. Quantum repeaters: The role of imperfect local operations in quantum communication. Phys. Rev. Lett. 81, 5932-5935 (1998).

31. Kómár, P. et al. A quantum network of clocks. Nature Phys. 10, 582-587 (2014)

32. Pelc, J., Langrock, C., Zhang, Q. \& Fejer, M. Influence of domain disorder on parametric noise in quasi-phase-matched quantum frequency converters. Opt. Lett. 35, 2804-2806 (2010).

33. Hayes, D. et al. Coherent error suppression in multiqubit entangling gates. Phys. Rev. Lett. 109, 020503 (2012).

\section{Acknowledgements}

We thank K. R. Brown, L-M. Duan, J. Kim, P. Kwiat, D. N. Matsukevich, P. Maunz, D. L. Moehring, S. Olmschenk and P. Richerme for helpful discussions. This work was supported by the Intelligence Advanced Research Projects Activity, the Army Research
Office MURI Program on Hybrid Quantum Optical Circuits, and the NSF Physics Frontier Center at JQI.

\section{Author contributions}

D.H., I.V.I., G.V., C.C., S.D., S.M.C. and C.M. all contributed to the experimental design, construction, data collection and analysis of this experiment. All authors contributed to this manuscript.

\section{Additional information}

Reprints and permissions information is available online at www.nature.com/reprints. Correspondence and requests for materials should be addressed to D.H.

\section{Competing financial interests}

The authors declare no competing financial interests. 